\title{
Congenital myasthenic syndrome with episodic apnoea: clinical, neurophysiological and genetic features in the long-term follow-up of 19 patients
}

\author{
Grace McMacken $^{1}\left[\right.$ - Roger G. Whittaker ${ }^{2} \cdot$ Teresinha Evangelista $^{1} \cdot$ Angela Abicht $^{3} \cdot$ Marina Dusl $^{3}$ (1) . \\ Hanns Lochmüller ${ }^{1}$ (i)
}

Received: 11 August 2017 / Revised: 19 November 2017 / Accepted: 20 November 2017 / Published online: 30 November 2017

(C) The Author(s) 2017. This article is an open access publication

\begin{abstract}
Background Congenital myasthenic syndrome with episodic apnoea (CMS-EA) is a rare but potentially treatable cause of apparent life-threatening events in infancy. The underlying mechanisms for sudden and recurrent episodesof respiratory arrest in these patients are unclear. Whilst CMS-EA is most commonly caused by mutations in CHAT, the list of associated genotypes is expanding.

Methods We reviewed clinical information from 19 patients with CMS-EA, including patients with mutations in CHAT, SLC5A7 and RAPSN, and patients lacking a genetic diagnosis.

Results Lack of genetic diagnosis was more common in CMS-EA than in CMS without EA (56\% $n=18$, compared to 7\% $n=97)$. Most patients manifested intermittent apnoea in the first 4 months of life $(74 \%, n=14)$. A degree of clinical improvement with medication was observed in most patients $(74 \%, n=14)$, but the majority of cases also showed a tendency towards complete remission of apnoeic events with age (mean age of resolution 2 years 5 months). Signs of impaired neuromuscular transmission were detected on neurophysiology studies in $79 \%(n=15)$ of cases, but in six cases, this was only apparent following specific neurophysiological testing protocols (prolonged high-frequency stimulation).

Conclusions A relatively large proportion of CMS-EA remains genetically undiagnosed, which suggests the existence of novel causative CMS genes which remain uncharacterised. In light of the potential for recurrent life-threatening apnoeas in early life and the positive response to therapy, early diagnostic consideration of CMS-EA is critical, but without specific neurophysiology tests, it may go overlooked.
\end{abstract}

Keywords Congenital myasthenic syndrome $\cdot$ Neuromuscular disease $\cdot$ Neurophysiology $\cdot$ Neuromuscular junction

\section{Background}

Congenital myasthenic syndromes (CMS) are a group of disorders caused by mutations in genes encoding proteins responsible for the function and integrity of the

Grace McMacken

grace.mcmacken@ncl.ac.uk

1 John Walton Muscular Dystrophy Research Centre, MRC Centre for Neuromuscular Diseases, Institute of Genetic Medicine, Newcastle University, Newcastle upon Tyne, UK

2 Institute of Neuroscience, Newcastle University, Newcastle upon Tyne, UK

3 Friedrich-Baur-Institute, Ludwig Maximilians University, Munich, Germany neuromuscular junction (NMJ), resulting in impairment of the safety margin necessary for reliable neuromuscular transmission. The first identified mutations were in genes encoding subunits of the acetylcholine receptors (AChRs), and these remain the most common subtypes of CMS worldwide today [1]. However, in recent years the discovery of CMSrelated genes has accelerated, and to date over 30 genes have been implicated (Fig. 1).

Although the clinical spectrum is increasingly diverse, CMS are characterised by fatigable weakness typically of early onset, a positive family history, and abnormal neurophysiology tests, namely repetitive nerve stimulation (RNS) or single-fibre EMG (SFEMG) [1]. In RNS, supramaximal electrical stimulation is delivered to a motor nerve via surface electrodes. In normal muscle, the resulting response (known as the compound muscle action potential or CMAP) 


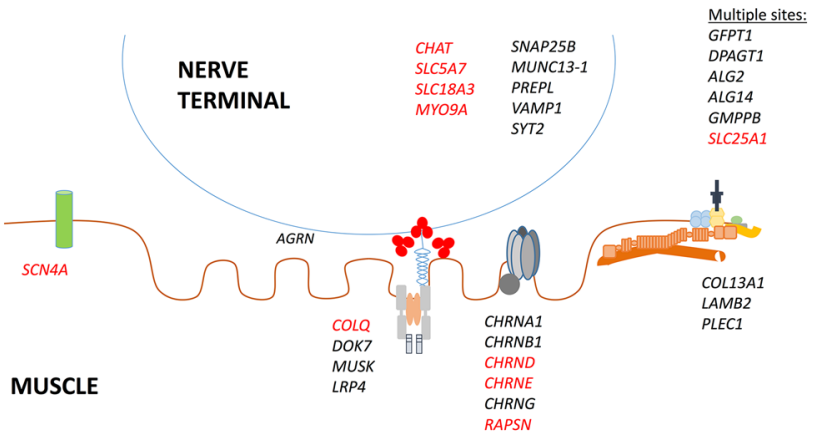

Fig. 1 Heterogeneity of genetic defects in CMS: Mutations are described in genes encoding pre-synaptic, synaptic and post-synaptic proteins, proteins of the extracellular matrix and dystrophinassociated glycoprotein complex and in ubiquitously expressed proteins involved in glycosylation (GFPT1, DPAGT1, ALG2, ALG14, $G H M P P B)$ and mitochondrial function (SLC25A1) which may act at multiple sites. Genes in which mutations have been previously described in patients with EA are highlighted in red

amplitude remains constant over a wide range of frequencies, since the safety margin for neuromuscular transmission is large. In patients with abnormalities of neuromuscular transmission however, the CMAP amplitude can vary with repetitive stimulation. In pre-synaptic disorders involving defects of neurotransmitter release, the baseline CMAP amplitude is characteristically small, with high-frequency nerve stimulation causing an incremental CMAP response as a result of calcium build-up in the pre-motor terminal. In contrast, in post-synaptic disorders the slight reduction in the release of the neurotransmitter acetylcholine (ACh) caused by low-frequency stimulation can cause the end-plate potential to fall below the threshold required to generate a muscle action potential in a proportion of the muscle fibres. This results in decrement of the CMAP amplitude. However, in many cases, whether the disorder is pre- or post-synaptic cannot be effectively differentiated using neurophysiological testing alone.

In CMS due to mutations in the gene encoding the presynaptic choline acetyltransferase (CHAT), the deficit is not in calcium-triggered ACh release, but in re-synthesis of $\mathrm{ACh}$ following re-uptake by the nerve terminal $[2,3]$. Since the terminal contains a large reserve pool of ACh vesicles, a decremental response on RNS is only apparent once this pool has been exhausted. Hence, low-frequency stimulation typically produces no decrement, with the abnormality only becoming apparent following prolonged high-frequency nerve stimulation (e.g. $10 \mathrm{~Hz}$ for $5 \mathrm{~min}$ ).

SFEMG determines the variability in the latency of neuromuscular transmission for individual muscle fibres within the same motor unit, or "jitter". Increased jitter is more sensitive for detecting impaired neuromuscular transmission than RNS, but is less specific since the findings are essentially identical for both pre- and post-synaptic disorders and hence must be interpreted along with clinical symptoms and signs.

CMS may be particularly difficult to diagnose in neonates, in whom non-specific features such as generalised hypotonia, arthrogryposis and poor suck or cry may be the only clinical signs. In this age group, certain subsets of CMS may be associated with life-threatening episodic apnoea (CMS-EA); a rare, but potentially treatable cause of apparent life-threatening events (ALTEs). Whilst CMSEA was initially described in association with mutations in CHAT [2], the genetic basis has expanded, and several more recently described CMS genes have been shown to be associated with EA (Table 1). Genetic diagnosis for these conditions is all the more imperative given that effective treatments may prevent these life-threatening crises, but treatment response varies depending on the genetic subtype. The underlying mechanism of EA in disorders of neuromuscular transmission is unknown. We reviewed the clinical course of 19 patients with CMS-EA, to demonstrate potential diagnostic pitfalls and to assess long-term prognosis.

\section{Methods}

Clinical information was reviewed from 34 patients who currently attend the CMS clinic at the John Walton Muscular Dystrophy Research Centre, Newcastle Upon Tyne, United Kingdom, and 929 patients from around the world who were referred to our laboratory for genetic testing between 1997 and 2011 in the context of a clinical suspicion of CMS following review by experienced neuromuscular clinicians [4]. Patients had convincing evidence of CMS on the basis of clinical features, neurophysiological studies, laboratory investigations (including measurement of AChR antibodies and serum creatine kinase) and, in many cases, a prior muscle biopsy. EA was noted as being present where the clinician had recorded recurrent periods of respiratory arrest during the disease course; patients described as having respiratory insufficiency (causing inadequate oxygenation) but lacking clear description of recurrent apnoeic events (with cessation of respiration) were not included. CMS-EA was clearly described in 32 patients; however, detailed clinical information and follow-up were available for 19 CMS-EA patients. For these cases, clinical, genetic and neurophysiological data were retrospectively reviewed. Genomic DNA samples or EDTA blood were provided to our laboratory from neuromuscular and neurology centres worldwide. Sanger-based mutation screening was carried out on a gene-after-gene basis according to phenotype. In one case, which has been previously described, the causative gene (MYO9A) was identified following whole exome analysis [5]. Informed 
Table 1 CMS genes associated with EA

\begin{tabular}{|c|c|c|}
\hline Gene & Protein and function & Clinical features \\
\hline CHAT & $\begin{array}{l}\text { Choline acetyltransferase (ChAT); re-synthesis of acetylcholine } \\
\text { (ACh) from choline and acetyl-CoA in pre-synaptic nerve } \\
\text { terminal [2] }\end{array}$ & $\begin{array}{l}\text { Can exhibit striking clinical variability both between and within } \\
\text { families. Positive response to AChEIs is seen in almost all } \\
\text { cases }[3,22]\end{array}$ \\
\hline$R A P S N$ & $\begin{array}{l}\text { Rapsyn; post-synaptic scaffolding protein, interacts with AChRs } \\
\text { to induce clustering [23] }\end{array}$ & $\begin{array}{l}\text { Two main phenotypes: late-onset with fatigable limb weakness, } \\
\text { early onset characterised by arthrogryposis, high-arched pal- } \\
\text { ate, and facial, cervical and bulbar weakness [24-26] }\end{array}$ \\
\hline $\begin{array}{l}\text { CHRNE } \\
\text { (fast } \\
\text { channel) }\end{array}$ & $\begin{array}{l}\text { Epsilon subunit of AChR, altered kinetic properties following } \\
\text { binding of ACh to receptor }[27,28]\end{array}$ & Severe weakness with crises [29] \\
\hline SLC5A7 & $\begin{array}{l}\text { High-affinity choline transporter } 1 \text {, resynthesises ACh in the pre- } \\
\text { synaptic nerve terminal [30] }\end{array}$ & $\begin{array}{l}\text { Phenotypes range from severe form with arthrogryposis, hypoto- } \\
\text { nia and early lethality, to neonatal onset CMS with prominent } \\
\text { EA [20] }\end{array}$ \\
\hline SLC18A3 & $\begin{array}{l}\text { Vesicular acetylcholine transporter (VAChT), uptake of ACh } \\
\text { into pre-synaptic vesicles [20] }\end{array}$ & $\begin{array}{l}\text { Ptosis, ophthalmoplegia and fatigability. Deterioration of symp- } \\
\text { toms in cold temperature described in one case [20] }\end{array}$ \\
\hline$C O L Q$ & $\begin{array}{l}\text { ColQ, collagenic tail of } \mathrm{AChE} \text { which anchors } \mathrm{AChE} \text { in the post- } \\
\text { synaptic membrane }[31,32]\end{array}$ & $\begin{array}{l}\text { Broad phenotype, from adult onset limb girdle CMS to early } \\
\text { onset severe and progressive forms. Slowing of the pupillary } \\
\text { light reflex ( } 25 \% \text { of cases). Worsening with AChEI therapy } \\
{[31,33]}\end{array}$ \\
\hline$C H R N D$ & Delta subunit of AChR & Phenotype overlapping with rapsyn-CMS [34-36] \\
\hline$S C N 4 A$ & $\begin{array}{l}\text { Voltage gated sodium channel (Nav1.4), influx of sodium ions } \\
\text { into post-synaptic membrane and generation of muscle action } \\
\text { potential }[37,38]\end{array}$ & $\begin{array}{l}\text { Relatively severe limb weakness, ophthalmoplegia and ptosis. } \\
\text { Apnoeic attacks which persisted from infancy into early adult- } \\
\text { hood are described [38] }\end{array}$ \\
\hline МYО9А & $\begin{array}{l}\text { Unconventional myosin Myo9A, presumed pre-synaptic function } \\
\text { [5] }\end{array}$ & $\begin{array}{r}\text { Identified in two families, neonatal onset EA which responded } \\
\text { dramatically to pyridostigmine and 3,4-DAP is described [5] }\end{array}$ \\
\hline
\end{tabular}

consent was obtained from all participants by local institutions. All genetically undiagnosed patients had been screened for mutations in CHAT, RAPSN, CHRNE, COLQ, DOK7, CHRNA1, CHRND, CHRNB1, SLC5A7 and SLC18A3. Neurophysiological assessment was performed at local institutions according to protocols described previously [6, 7]. Decrement was defined as a decrease in CMAP amplitude of $10 \%$ or more between the first and fourth CMAPs.

\section{Results}

Episodic apnoea was described in 3\% $(n=32 / 963)$ of CMS cases. Of the CMS-EA cases, $44 \%(n=14 / 32)$ were genetically diagnosed, compared with $93 \%(n=866 / 963)$ of all CMS cases. The most common causative mutations were in the CHAT gene (16\% of CMS-EA cases), with mutations in COLQ (9\%), RAPSN (6\%), SLC5A7 (6\%), MYO9A (3\%) and CHRNE (3\%) being causative for the remainder of the cases with genetic diagnoses (Fig. 2).

In 13 cases, there was insufficient information on long-term follow-up and these were excluded from further analysis. Clinical features, neurophysiology and management are described, therefore, for the remaining 19 cases (Table 2).

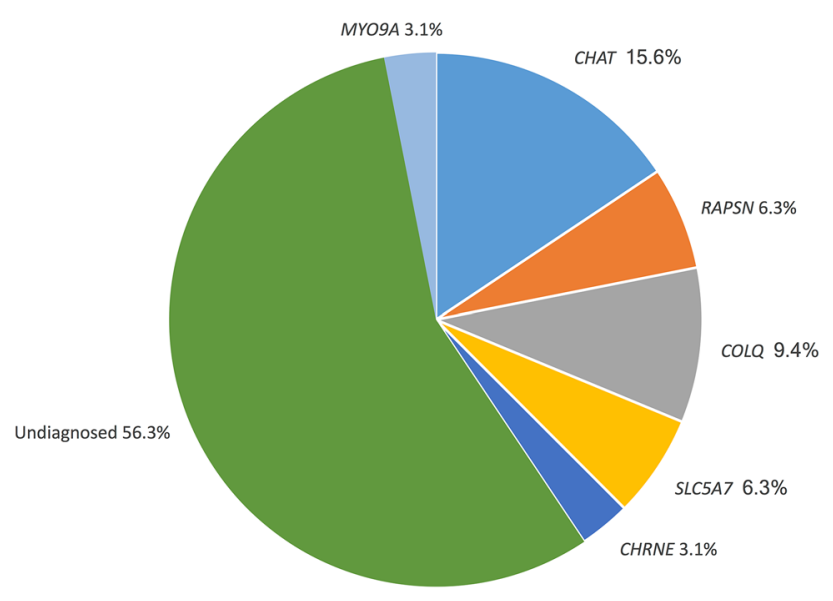

Fig. 2 Proportion of CMS-EA subtypes in our patient cohort $(n=32)$

\section{Clinical features}

All cases were proband, apart from cases 14 and 15 who are siblings. There was no family history of a neuromuscular disorder in any case, and consanguinity was reported in only two families. The disease manifested at birth in $84 \%$ ( $n=16$ ) of cases, with the remaining three cases presenting within the first 2 months of life. Signs present at birth were hypotonia (16 cases) and arthrogryposis (4 cases). 


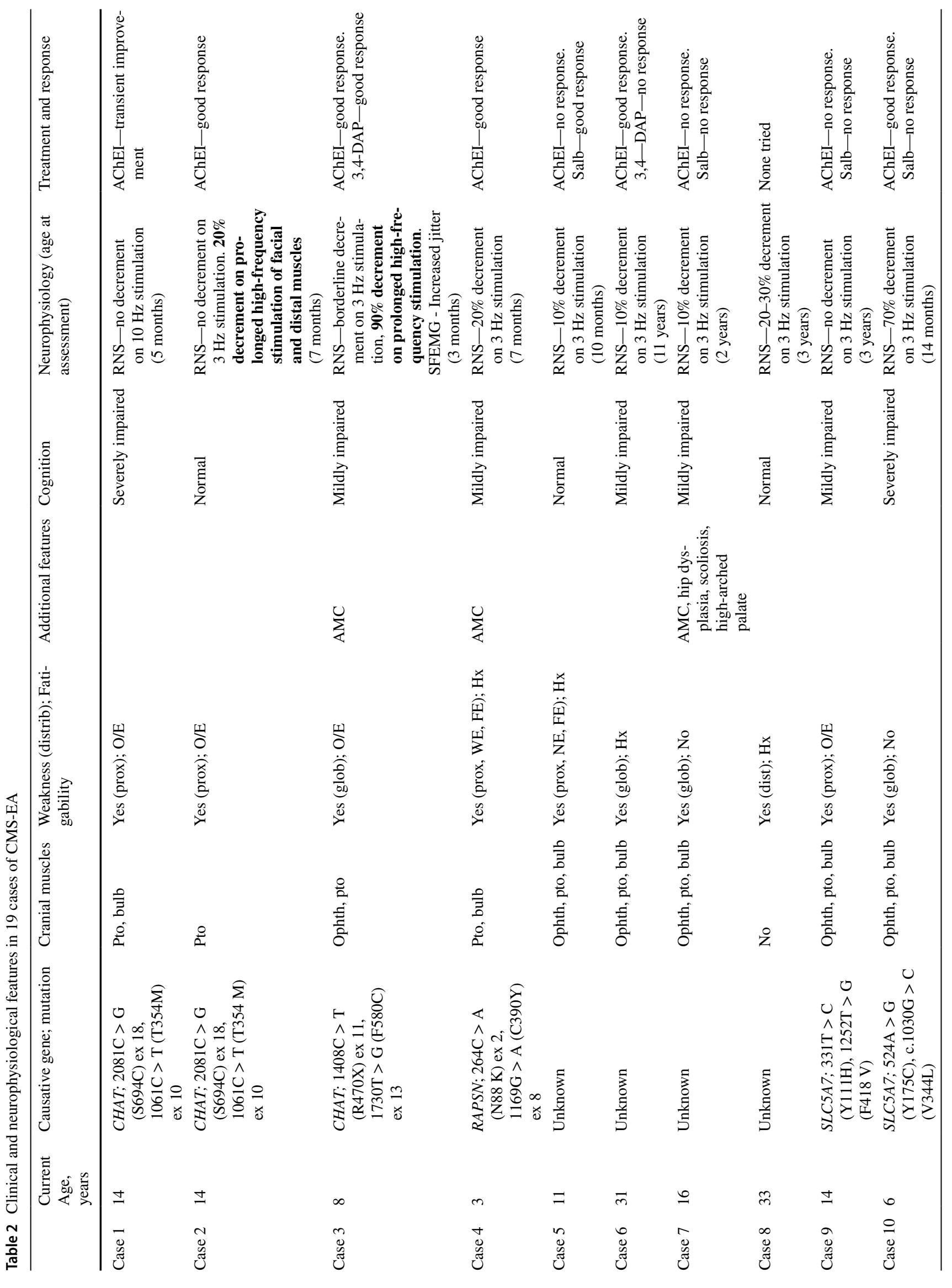


198

Journal of Neurology (2018) 265:194-203




Antenatal complications were recognised in $42 \%(n=8)$ of patients, and included reduced foetal movements and/or polyhydramnios.

Age of onset of EA was variable. Most patients had their first apnoea in the hours following birth $(n=5,26 \%)$ or in the first 4 weeks of life $(n=5,26 \%)$. For four cases (21\%), the first apnoeic event occurred between the age of 1-4 months, and four cases developed EA between 4 and 12 months of age. In one case, EA did not occur until the age of 18 months.

Cognitive development was abnormal in nine cases (47\%); in three cases, this was severe and considered to be secondary to hypoxic brain damage. Brain MRI was available for ten patients, and described as normal in seven, with two cases showing features in-keeping with hypoxic ischaemic encephalopathy, and one case (with CHAT-CMS) showing hypoplasia of the cerebellar vermis.

Median diagnostic delay from first symptoms to the clinical diagnosis of CMS (prior to genetic confirmation) was 8 months (range 1-96 months). Differential diagnoses included laryngomalacia, epilepsy, cardiac conduction defects, congenital hypothyroidism, Prader-Willi syndrome and spinal muscular atrophy.

\section{Apnoeic events and respiratory function}

Infections, including respiratory tract infections, were the most common precipitating factor for apnoeas, reported in $74 \%(n=14)$ of patients (Table 3$)$. Apnoeas were also reported during feeding, stress, crying, increased activity and increased environmental temperature. Frequency of apnoeas varied significantly, independent of genotype, from approximately one episode per month, to very frequent episodes occurring up to 40 times per day.

During apnoeic episodes, myasthenic features typically worsened, with worsening of hypotonia, bulbar weakness and ptosis reported in 14, 6 and 4 cases, respectively. Approximate duration of events ranged from $30 \mathrm{~s}$ to over 30 min (persisting until the patient was intubated and ventilated, and resulting in permanent brain damage), but mean duration of a typical event was $2 \mathrm{~min}$.

There was significant morbidity and mortality in the cohort, with two patients having died following prolonged apnoeas, aged 11 months (genetically undiagnosed) and 3 years (with RAPSN mutations), respectively, and a further case with severe hypoxic brain injury being permanently ventilated (SLC5A7 mutations). However, overall progressive improvement and tendency towards resolution of apnoeic episodes was reported in the majority of cases (Fig. 3). For 11 cases there was complete remission of apnoeas (mean age of resolution 2 and 5 months). A further five cases showed tendency towards remission but still experienced apnoeas during infections. 
There was no genotype-phenotype correlation between cases who had remission of EA and those who still experienced apnoeas.

\section{Neurophysiology}

All patients had neurophysiological assessment; 4 cases had RNS and SFEMG, and 15 cases had been investigated using RNS alone. Abnormal RNS was seen in 15/19 cases (79\%); in four cases, in whom only RNS was performed, no decrement was detected. In 5 of the 15 cases with abnormal RNS, stimulation at $3 \mathrm{~Hz}$ was borderline or negative, but prolonged high-frequency RNS $(10 \mathrm{~Hz}$ for $5 \mathrm{~min})$ revealed marked decrement. In a further patient (genetically undiagnosed) increment was detected on prolonged stimulation. All cases who had SFEMG in addition to RNS demonstrated increased jitter.

\section{Management}

18 cases had been treated with acetylcholinesterase inhibitors (AChEIs) at some point. Of these, $67 \%(n=12)$ had a sustained clinical improvement, which included all patients with mutations in RAPSN and CHAT, one patient with $S L C 5 A 7$ mutations and seven patients who were genetically undiagnosed. Transient improvement but subsequent deterioration following commencement of AChEIs was seen in two patients (both genetically undiagnosed). AChEIs had no effect in four cases (three genetically undiagnosed, and one SLC5A7-CMS). The $\beta_{2}$-agonist salbutamol resulted in clinical improvement in three cases (genetically undiagnosed, fast channel syndrome and CHAT-CMS), transient improvement in one case (undiagnosed) and no response in the remaining three cases (one undiagnosed, and two with SLC5A7-CMS). 3,4-Diaminopyridine (3,4-DAP) was used as an adjunctive therapy in two cases, resulting in improvement in one (with CHAT-CMS) and no response in the other (genetically undiagnosed). All six cases in whom abnormal RNS had only been detected following prolonged high-frequency stimulation had clinical benefit from AChEI therapy.

Most patients $(n=14,74 \%)$ had normal respiratory function between apnoeic events. Three cases required nocturnal non-invasive ventilation (one with CHAT-CMS and two genetically undiagnosed cases), and one was permanently ventilated via tracheostomy. Hospital attendances were common, with $41 \%(n=8)$ of cases requiring frequent $(>1$ per year) admissions. Fourteen patients had required ventilation during periods of respiratory crisis (11 invasive and 3 noninvasive ventilation).

Table 3 Apnoeic events and respiratory function in our patient cohort

\begin{tabular}{|c|c|c|c|c|}
\hline & Precipitating events & Associated symptoms during apnoea & Recurrent LRTI & $\begin{array}{l}\text { Respiratory function } \\
\text { between episodes }\end{array}$ \\
\hline Case 1 & Feeding, infection, stress & Hypotonia, bulbar weakness, ptosis & Yes & Normal \\
\hline Case 2 & Feeding, infection, stress & Hypotonia & Previously, now resolved & Normal \\
\hline Case 3 & Infection & Hypotonia & Previously, now resolved & Normal \\
\hline Case 4 & Infection, feeding & Hypotonia & Yes & Normal \\
\hline Case 5 & Infection, during sleep & Hypotonia, bulbar weakness & Yes & Normal \\
\hline Case 6 & Stress, infection, higher temperature & Ptosis, bulbar weakness, hypotonia & Previously, now resolved & Normal \\
\hline Case 7 & Crying & Hypotonia & No & Normal \\
\hline Case 8 & Infection & None noted & Previously, now resolved & Normal \\
\hline Case 9 & Feeding, infection & Ptosis, bulbar weakness, hypotonia & No & Normal \\
\hline Case 10 & Infection & Hypotonia & Yes & Permanently ventilated \\
\hline Case 11 & Infection, feeding, crying & None noted & Yes & Normal \\
\hline Case 12 & Feeding, infection & Hypotonia, facial weakness & Yes & Impaired \\
\hline Case 13 & Feeding & No & Yes & Normal \\
\hline Case 14 & Feeding & Hypotonia, ptosis, cyanosis & Yes & Not assessed \\
\hline Case 15 & Infection, increased activity, sleep & Hypotonia & Yes & Normal \\
\hline Case 16 & Infection, increased activity, sleep & Hypotonia, bulbar weakness & Yes & Normal \\
\hline Case 17 & Feeding & Hypotonia & No & Normal \\
\hline Case 18 & Feeding, infection, during sleep & Hypotonia, cyanosis & Yes & Impaired \\
\hline Case 19 & Infection, feeding & Hypotonia, cyanosis & Yes & Impaired \\
\hline
\end{tabular}

LRTI lower respiratory tract infection 


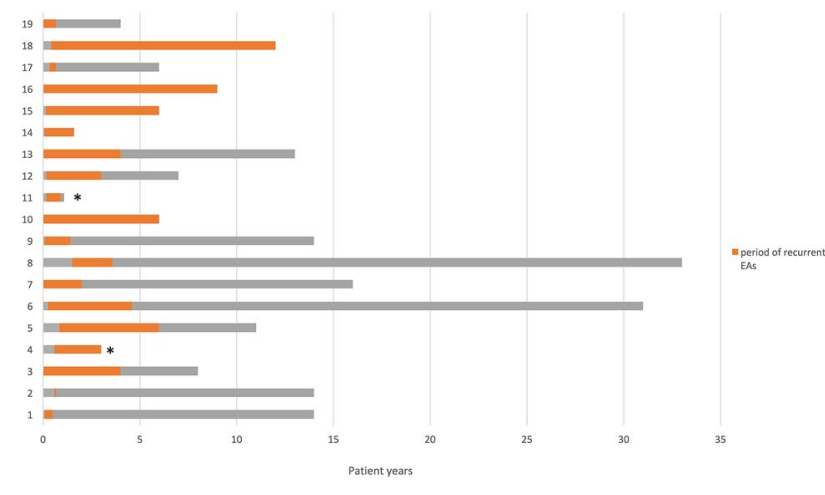

Fig. 3 Resolution of apnoeic events over time: For the majority of cases, the period of recurrent EAs (orange) during a patient's life span (gray) began in the first months of life and resolved in early childhood. Cases marked $*$ are deceased

\section{Discussion}

Apparent life-threatening events (ALTEs), defined as episodes characterised by some combination of apnoea, skin colour change, change in muscle tone and choking or gagging [8], have an extremely broad differential diagnosis. Causes include epilepsy, cardiac arrhythmia or structural cardiac defects, gastro-oesophageal reflux disease, respiratory tract infections and upper airway obstruction [9-12]. ALTEs are most common in the first 10 weeks of life, with cyanosis and apnoea often being the most prominent or only symptoms [11]. CMS is rarely considered as a possible differential diagnosis for ALTEs, thus many patients undergo extensive investigation before the possibility is considered. In our cohort, average diagnostic delay was substantial at 8 months. Worsening of myasthenic symptoms during apnoeas was frequently observed, and these features may provide a diagnostic clue. In practice, however, many patients may be admitted directly to intensive care units and may not be examined by a paediatric neurologist or neuromuscular specialist during these crises.

In early infancy, abnormal neurophysiology tests may be the only indication of a NMJ disorder. However, we observed in this cohort that without specific neurophysiological assessment, a pre-synaptic abnormality may go overlooked. In $32 \%(n=6)$ of cases, no abnormality was observed at low-frequency RNS, with decrement or increment only becoming apparent following prolonged highfrequency stimulation. In two of these cases, the genetic diagnosis was of ChAT deficiency, in which re-synthesis of $\mathrm{ACh}$ is known to be impaired. In the other four cases, the genetic diagnosis was unknown, but it may be that these also affect proteins involved in this pathway. Failure to perform this additional test could result in the diagnosis of CMS-EA being discounted. Furthermore, all of these cases showed a positive response to AChEI therapy. Such prolonged studies can be uncomfortable for the patient, and may require sedation. Nevertheless, given the risk of fatal apnoeas if the diagnosis is missed, and the potential for clinical improvement with AChEIs, these prolonged studies should be performed if deficiency of ACh re-synthesis is suspected.

The underlying mechanism(s) giving rise to these sudden and recurrent apnoeas is yet to be characterised. Respiratory control mechanisms respond to input from neural and chemical receptors, which are integrated by the respiratory centres in the medulla and pons. These subsequently provide neuronal drive to respiratory muscles, maintaining upper airway patency and determining the level of ventilation. Recurrent apnoea could be due to an abnormality at any point along this axis. The majority of mutations causing CMSEA lead to pre-synaptic defects (Fig. 1), which have important functions for both central and neuromuscular synaptic function. This, along with the tendency for patients to have normal respiratory function between apnoeas, could point to a centrally mediated mechanism. In addition, the proportion of patients with impaired cognitive function in our cohort $(47 \%)$ was higher than expected and is not something that has been previously well-characterised in disorders of the NMJ. Any impairment of higher function in CMS-EA patients is often explained by hypoxic brain damage due to repeated respiratory failure, although the possibility of impaired CNS maturation due to reduced neurotransmitter in central cholinergic neurons has not been fully explored. The most common CMS-EA gene, CHAT, has important functions in both central and peripheral synapses, and deficiency of ChAT has been reported in Alzheimer's disease, idiopathic Parkinson's disease, Huntington's disease and schizophrenia [13-16]. Furthermore, in cases of sudden infant death syndrome, decreased activity of ChAT has been demonstrated in the CNS $[17,18]$. Cognitive and behavioural have also been described in the recently identified CMS subtype due to mutations in SLC5A7, which encodes the high-affinity choline transporter 1 , necessary for uptake of choline from the synaptic space for the synthesis of $\mathrm{ACh}$ at central and peripheral cholinergic synapses [19]. A further CMS-EA gene involved in ACh release, SLC18A3, encodes the vesicular acetylcholine transporter (VAChT), which mediates the vesicular storage of ACh in the central and peripheral nervous system. SLC18A3 mutations are associated with a severe CMS phenotype and learning difficulties are also described [20,21]. The CMS-EA genes SNAP25B, $V A M P 1$ and MUNC13 encode proteins of the SNARE complex. These are essential for calcium-triggered exocytosis at central and neuromuscular synapses; therefore, it is unsurprising that CMS due to mutations in these genes may be associated with epilepsy and impaired brain development $[22,23]$. Abnormalities of the CNS have also been reported in CMS due to mutations in the unconventional myosin gene 
MYO9A. MYO9A also appears to have pre-synaptic function and localization at the NMJ, although the mechanism for NMJ dysfunction in these patients is yet to be determined [5].

Administration of one or more drugs is indicated once the diagnosis of CMS-EA is established. Several drugs have shown convincingly positive effects in reducing the frequency of apnoeas, including AChEIs, 3,4-DAP and sympathomimetics (ephedrine or salbutamol). Because of the sudden nature of these attacks, parents should be provided with an inflatable rescue bag and fitted mask, trained in CPR and may be provided with a home apnoea monitor. Parents may also be instructed to give additional AChEI doses during infection or other potential precipitating events.

We recognise that this study has several limitations. We include a small number of patients, contributed to by the rarity of the condition. Data were collected retrospectively and over a long period of time (1997-2011) during which the awareness of CMS and availability for genetic testing has increased. However, even in this patient cohort, who continues to be tested for new causative CMS genes as they are discovered, a relatively large proportion $(56 \%)$ of cases remain genetically undiagnosed. CMS-EA is a challenging diagnosis, with several mimics, and this proportion could suggest an alternative diagnosis other than a neuromuscular transmission defect in these patients. However, it may also reflect further CMS causing genes which have not yet been discovered.

Given the likelihood of recurring episodes, the potential for psychomotor impairment due to secondary hypoxic brain damage, and the positive effect of available treatments, CMS-EA is an important diagnosis not to miss. Prompt diagnosis and initiation of treatment and ventilatory support is imperative in early life, when EA may be frequent, but the majority of cases will exhibit a tendency towards remission. Improved understanding of the mechanism of this condition may provide insights into further causative genes for genetically undiagnosed patients, and lead to improved diagnostic and treatment strategies.

Acknowledgements We thank the following healthcare professionals for the provision of clinical information and patient samples: Dr Sigrid Lyding, University of Bonn Centre for Paediatrics, Germany; Dr Ulrike Schara, Department of Paediatric Neurology, Universitätsklinikum, Essen, Germany; Dr Elena Pegoraro, Department of Neurosciences, University of Padova, Italy; Dr Maja von der Hagen, Department of Neuropathology, University Hospital Carl Gustav Carus, Dresden, Germany; Dr Robertino Dilena, Service of Pediatric Epileptology-Unit of Clinical Neurophysiology, Fondazione IRCCS Ca' Granda, Ospedale Maggiore Policlinico, Milan, Italy; Dr Uluç Yiş, Dokuz Eylül University Faculty of Medicine Department of Child Health and Diseases, Izmir, Turkey; Dr Roseli Gomes, Hospital Pedro Hispano, Matosinhos, Portugal; Dr Monica Troncoso, Servicio Neurologico Infantil, Hospital Clinico San Borja Arriaran, Santa Rosa 1234, Santiago, Chile; Dr Andrés Nascimento Osorio and Dr Jauma Colomer, Unidad de patología neuromuscular, Hospital Sant Joan de Déu, Barcelona;
Prof Eugen Boltshauser and Dr Andrea Klein, Department of Pediatric Neurology, University Children's Hospital, Zurich, Switzerland; Dr Kristina LAH Tomulic, Pediatric Intensive Care Unit, University Hospital Rijeka; Dr Bulent Kara and Dr Sevil Gökulut, Department of Pediatrics, Division of Child Neurology, Kocaeli University, Kocaeli, Turkey; Dr Guja Astrea, Department of Developmental Neuroscience, IRCCS Stella Maris Foundation, Pisa, Italy; Dr Federica Ricci, Center for Neuromuscular Diseases, Regina Margherita Children Hospital, and Department of Neurosciences, University of Torino, Italy; Dr Chiara Fiorrillo, Molecular Medicine, IRCCS Fondazione Stella Maris, Pisa, Italy. GM receives funding from the Association of British Neurologists and Guarantors of Brain Clinical Research Training Fellowship, and the Wellcome Trust Pathfinder Award. HL receives funding from the Medical Research Council as part of the MRC Centre for Neuromuscular Diseases (reference G1002274, Grant ID 98482), and by the European Union Seventh Framework Programme (FP7/20072013) under Grant agreement No. 305444 (RD-Connect) and 305121 (NeurOmics).

\section{Compliance with ethical standards}

Ethical standards All human studies have been approved by the appropriate ethics committee (Newcastle and North Tyneside 1 Research Ethics Committee) and have, therefore, been performed in accordance with the ethical standards laid down in the 1964 Declaration of Helsinki and its later amendments.

Conflicts of interest The authors declare that they have no conflict of interest.

Open Access This article is distributed under the terms of the Creative Commons Attribution 4.0 International License (http://creativecommons.org/licenses/by/4.0/), which permits unrestricted use, distribution, and reproduction in any medium, provided you give appropriate credit to the original author(s) and the source, provide a link to the Creative Commons license, and indicate if changes were made.

\section{References}

1. Abicht A, Müller J, Lochmüller H (1993) Congenital myasthenic syndromes. In: Pagon RA, Adam MP, Ardinger HH et al (eds) GeneReviews $\left({ }^{\circledR}\right)$. University of Washington, Seattle

2. Ohno K, Tsujino A, Brengman JM et al (2001) Choline acetyltransferase mutations cause myasthenic syndrome associated with episodic apnea in humans. Proc Natl Acad Sci USA 98:20172022. https://doi.org/10.1073/pnas.98.4.2017

3. Arredondo J, Lara M, Gospe SM et al (2015) Choline acetyltransferase mutations causing congenital myasthenic syndrome: molecular findings and genotype-phenotype correlations. Hum Mutat 36:881-893. https://doi.org/10.1002/humu.22823

4. Abicht A, Dusl M, Gallenmüller C et al (2012) Congenital myasthenic syndromes: achievements and limitations of phenotypeguided gene-after-gene sequencing in diagnostic practice: a study of 680 patients. Hum Mutat 33:1474-1484. https://doi. org/10.1002/humu.22130

5. O'Connor E, Töpf A, Müller JS et al (2016) Identification of mutations in the MYO9A gene in patients with congenital myasthenic syndrome. Brain 139:2143-2153. https://doi.org/10.1093/ brain/aww 130

6. Kennett RP, Fawcett PR (1993) Repetitive nerve stimulation of anconeus in the assessment of neuromuscular transmission disorders. Electroencephalogr Clin Neurophysiol 89:170-176 
7. Benatar M, Hammad M, Doss-Riney H (2006) Concentric-needle single-fiber electromyography for the diagnosis of myasthenia gravis. Muscle Nerve 34:163-168. https://doi.org/10.1002/ mus. 20568

8. American Academy of Pediatrics (1987) National Institutes of Health Consensus Development Conference on Infantile Apnea and Home Monitoring Sept 29 to Oct 1, 1986. Pediatrics 79:292-299

9. Keeton BR, Southall E, Rutter N et al (1977) Cardiac conduction disorders in six infants with "near-miss" sudden infant deaths. Br Med J 2:600-601

10. Rahilly PM (1991) The pneumographic and medical investigation of infants suffering apparent life threatening episodes. J Paediatr Child Health 27:349-353

11. Davies F, Gupta R (2002) Apparent life threatening events in infants presenting to an emergency department. Emerg Med J EMJ 19:11-16. https://doi.org/10.1136/emj.19.1.11

12. Arens R, Gozal D, Williams JC et al (1993) Recurrent apparent life-threatening events during infancy: a manifestation of inborn errors of metabolism. J Pediatr 123:415-418. https://doi. org/10.1016/S0022-3476(05)81747-0

13. Baskin DS, Browning JL, Pirozzolo FJ et al (1999) Brain choline acetyltransferase and mental function in Alzheimer disease. Arch Neurol 56:1121-1123

14. Aquilonius SM, Eckernås SA, Sundwall A (1975) Regional distribution of choline acetyltransferase in the human brain: changes in Huntington's chorea. J Neurol Neurosurg Psychiatr 38:669-677

15. Mattila PM, Röyttä M, Lönnberg P et al (2001) Choline acetyltransferase activity and striatal dopamine receptors in Parkinson's disease in relation to cognitive impairment. Acta Neuropathol (Berl) 102:160-166

16. Karson CN, Casanova MF, Kleinman JE, Griffin WS (1993) Choline acetyltransferase in schizophrenia. Am J Psychiatry 150:454 459. https://doi.org/10.1176/ajp.150.3.454

17. Kinney HC, Filiano JJ, Harper RM (1992) The neuropathology of the sudden infant death syndrome. A review. J Neuropathol Exp Neurol 51:115-126

18. Oda Y (1999) Choline acetyltransferase: the structure, distribution and pathologic changes in the central nervous system. Pathol Int 49:921-937

19. Bauché S, O'Regan S, Azuma Y et al (2016) Impaired presynaptic high-affinity choline transporter causes a congenital myasthenic syndrome with episodic apnea. Am J Hum Genet. https://doi. org/10.1016/j.ajhg.2016.06.033

20. O'Grady GL, Verschuuren C, Yuen M et al (2016) Variants in SLC18A3, vesicular acetylcholine transporter, cause congenital myasthenic syndrome. Neurology. https://doi.org/10.1212/ WNL.0000000000003179

21. Aran A, Segel R, Kaneshige K et al (2017) Vesicular acetylcholine transporter defect underlies devastating congenital myasthenia syndrome. Neurology 88:1021-1028. https://doi.org/10.1212/ WNL.0000000000003720

22. Engel AG, Selcen D, Shen X-M et al (2016) Loss of MUNC13-1 function causes microcephaly, cortical hyperexcitability, and fatal myasthenia. Neurol Genet 2. https://doi.org/10.1212/ NXG.0000000000000105

23. Shen X-M, Selcen D, Brengman J, Engel AG (2014) Mutant SNAP25B causes myasthenia, cortical hyperexcitability, ataxia, and intellectual disability. Neurology 83:2247-2255. https://doi. org/10.1212/WNL.0000000000001079
24. Burke G, Cossins J, Maxwell S et al (2003) Rapsyn mutations in hereditary myasthenia: distinct early- and late-onset phenotypes. Neurology 61:826-828

25. Natera-de Benito D, Bestué M, Vilchez JJ et al (2016) Long-term follow-up in patients with congenital myasthenic syndrome due to RAPSN mutations. Neuromuscul Disord 26:153-159. https:// doi.org/10.1016/j.nmd.2015.10.013

26. Muller J, Abicht A, Burke G et al (2004) The congenital myasthenic syndrome mutation RAPSN N88K derives from an ancient Indo-European founder. J Med Genet 41:e104. https:// doi.org/10.1136/jmg.2004.021139

27. Ohno K, Wang H-L, Milone M et al (1996) Congenital myasthenic syndrome caused by decreased agonist binding affinity due to a mutation in the acetylcholine receptor $\varepsilon$ subunit. Neuron 17:157170. https://doi.org/10.1016/S0896-6273(00)80289-5

28. Sine SM (2012) End-plate acetylcholine receptor: structure, mechanism, pharmacology, and disease. Physiol Rev 92:1189-1234. https://doi.org/10.1152/physrev.00015.2011

29. Webster R, Liu W-W, Chaouch A et al (2014) Fast-channel congenital myasthenic syndrome with a novel acetylcholine receptor mutation at the $\alpha-\varepsilon$ subunit interface. Neuromuscul Disord 24:143-147. https://doi.org/10.1016/j.nmd.2013.10.009

30. Barwick KES, Wright J, Al-Turki S et al (2012) Defective presynaptic choline transport underlies hereditary motor neuropathy. Am J Hum Genet 91:1103-1107. https://doi.org/10.1016/j. ajhg.2012.09.019

31. Mihaylova V, Müller JS, Vilchez JJ et al (2008) Clinical and molecular genetic findings in COLQ-mutant congenital myasthenic syndromes. Brain J Neurol 131:747-759. https://doi. org/10.1093/brain/awm325

32. Ohno K, Brengman J, Tsujino A, Engel AG (1998) Human endplate acetylcholinesterase deficiency caused by mutations in the collagen-like tail subunit (ColQ) of the asymmetric enzyme. Proc Natl Acad Sci 95:9654-9659

33. Wargon I, Richard P, Kuntzer T, et al (2012) Long-term followup of patients with congenital myasthenic syndrome caused by COLQ mutations. Neuromuscul Disord 22:318-324. https://doi. org/10.1016/j.nmd.2011.09.002

34. Gomez CM, Maselli RA, Vohra BPS et al (2002) Novel delta subunit mutation in slow-channel syndrome causes severe weakness by novel mechanisms. Ann Neurol 51:102-112

35. Shen XM, Okuno T, Milone M, Otsuka K, Takahashi K, Komaki H, Giles E, Ohno K, Engel AG (2016) Mutations causing slowchannel myasthenia reveal that a valine ring in the channel pore of muscle AChR is optimized for stabilizing channel gating. Hum Mutat 37(10):1051-1059. https://doi.org/10.1002/humu.23043

36. Müller JS, Baumeister SK, Schara U et al (2006) CHRND mutation causes a congenital myasthenic syndrome by impairing coclustering of the acetylcholine receptor with rapsyn. Brain J Neurol 129:2784-2793. https://doi.org/10.1093/brain/awl188

37. Arnold WD, Feldman DH, Ramirez S et al (2015) Defective fast inactivation recovery of Nav1.4 in congenital myasthenic syndrome. Ann Neurol 77:840-850. https://doi.org/10.1002/ ana.24389

38. Tsujino A, Maertens C, Ohno K et al (2003) Myasthenic syndrome caused by mutation of the SCN4A sodium channel. Proc Natl Acad Sci 100:7377-7382. https://doi.org/10.1073/ pnas. 1230273100 